\title{
Finite Difference Scheme for Solving Parabolic Partial Differential Equations with Random Variable Input under Mean Square Sense
}

\author{
M. A. Sohaly and W. W. Mohammed \\ Mathematics Department, Faculty of Science, Mansoura University, Egypt.
}

Received: February 27, 2016 / Accepted: March 25, 2016 / Published: July 25, 2016.

\begin{abstract}
This study deal with seven points finite difference method to find the approximation solutions in the area of mean square calculus solutions for linear random parabolic partial differential equations. Several numerical examples are presented to show the ability and efficiency of this method.
\end{abstract}

Keywords: Mean Square Convergence, Random Partial Differential Equations, Finite Difference Technique.

\section{Introduction}

The differential problems in both partial and ordinary are powerful implement for describing the view of many applications in our life. The inputs like as functions or factors appearing in the applied models are subject to uncertainty, this reason may be from error measurements, or from the methods that used for modeling [1-7]. These objects drive us to agent to random models where they are containing random variables and stochastic processes. The data (initial conditions, source term or coefficients) are input by means of randomness [8-13]. The random difference method by using three and five points for the problem in the following type

$$
\begin{gathered}
u_{t}=\beta u_{x x}(x, t), t \in[0, T], x \in[0, X], \beta(r . v .) \\
u(x, 0)=u_{0}(x) \\
u(0, t)=u(X, t)=0, \beta \text { is a random variable. }
\end{gathered}
$$

Our aim of this work is to approximate the solution of equation (1) by using the seven points difference technique. This paper is organized as follows. Section 2 deals with some preliminary definitions. Section 3 is

Corresponding author: M. A. Sohaly, Mathematics Department, Faculty of Science, Mansoura University, Egypt. E-mail: m_stat2000@yahoo.com. addressed to the presentation and the proof of the convergence for the seven points difference scheme for solving equation (1) in mean square sense. In Section 4, the statistical mean value for the exact, and numerical solutions is obtained. Finally, we state the conclusions of this paper.

\section{Preliminaries}

The random difference technique $L_{k}^{n} u_{k}^{n}=G_{k}^{n}$ that according to the RPDE $L v=G$ satisfy the consistency under the mean square when the time $t=(n+1) \Delta t$, if for any smooth function $\Phi=$ $\Phi(x, t)$, we have in mean square:

$$
E\left|(L \Phi-G)_{k}^{n}-\left(L_{k}^{n} \Phi(k \Delta x, n \Delta t)-G_{k}^{n}\right)\right|^{2} \rightarrow 0
$$

At:

$$
\begin{gathered}
k \rightarrow \infty, n \rightarrow \infty, \Delta x \rightarrow 0, \Delta t \rightarrow \\
0 \text { and }(k \Delta x, n \Delta t) \rightarrow(x, t) .
\end{gathered}
$$

The random difference technique $L_{k}^{n} u_{k}^{n}=G_{k}^{n}$ that according to the RPDE $L v=G$, satisfy the exponential stability under the mean square, if for the positive constants $\varepsilon, \delta$, $\mathrm{k}$ also, negative' constant $\mathrm{b}$ such that:

$$
E\left|u_{k}^{n+1}\right|^{2} \leq k e^{b t} E\left|u^{0}\right|^{2},
$$

For ,all: $0 \leq t=(n+1) \Delta t, 0 \leq \Delta x \leq \varepsilon$ and $0 \leq$ $\Delta t \leq \delta$. 
The random difference technique $L_{k}^{n} u_{k}^{n}=G_{k}^{n}$, approximating RPDE $L v=G$ is convergent in the mean square at time $t=(n+1) \Delta t$, if

$$
E\left|u_{k}^{n}-u\right|^{2} \rightarrow 0
$$

At: $\Delta x \rightarrow 0, \Delta t \rightarrow 0, k \Delta x \rightarrow x, n \Delta t \rightarrow t$.

\section{Random Differential Scheme with Seven Points}

The difference scheme for equation (1) with seven points is]

$$
\begin{gathered}
u_{k}^{n+1}=(1-490 r \beta) u_{k}^{n}+r \beta\left[2 u_{k-3}^{n}-27 u_{k-2}^{n}+270 u_{k-1}^{n}+270 u_{k+1}^{n}-27 u_{k+2}^{n}+2 u_{k+3}^{n}\right] \\
u_{\mathrm{k}}^{0}=\mathrm{u}_{0}\left(\mathrm{x}_{\mathrm{k}}\right), \mathrm{u}_{0}^{\mathrm{n}}=\mathrm{u}_{\mathrm{x}}^{\mathrm{n}}=0, \beta, \text { is a random variable }
\end{gathered}
$$

where $u_{t}=\frac{u_{k}^{n+1}-u_{k}^{n}}{\Delta t}$, and

$$
u_{x x}=\frac{2 u_{k-3}^{n}-27 u_{k-2}^{n}+270 u_{k-1}^{n}-490 u_{k}^{n}+270 u_{k+1}^{n}-27 u_{k+2}^{n}+2 u_{k+3}^{n}}{180 \Delta x^{2}}
$$

\subsection{Consistency of RFDS (2)}

Theorem (1). The stochastic difference scheme with seven points (2) is consistent in mean square sense. As:

$$
\Delta t \rightarrow 0, \Delta x \rightarrow 0, n \rightarrow \infty, k \rightarrow \infty, \frac{k^{2}}{n} \rightarrow 0
$$

and

$$
(k \Delta x, n \Delta t) \rightarrow(x, t)
$$

Proof:

Assume that $\Phi(x, t)$ be a smooth function then:

and

$$
L(\Phi)_{k}^{n}=\Phi(k \Delta x,(n+1) \Delta t)-\Phi(k \Delta x, n \Delta t)-\beta \int_{n \Delta t}^{(n+1) \Delta t} \Phi_{x x}(k \Delta x, s) d s
$$

$$
\begin{aligned}
L_{k}^{n} \Phi=\Phi(k \Delta x, & (n+1) \Delta t)-\Phi(k \Delta x, n \Delta t) \\
& -r \beta(2 \Phi((k-3) \Delta x, n \Delta t)-27 \Phi((k-2) \Delta x, n \Delta t)+270 \Phi((k-1) \Delta x, n \Delta t) \\
& -490 \Phi(k \Delta x, n \Delta t)+270 \Phi((k+1) \Delta x, n \Delta t)-27 \Phi((k+2) \Delta x, n \Delta t) \\
& +2 \Phi((k+3) \Delta x, n \Delta t))
\end{aligned}
$$

Then we have:

$$
\begin{aligned}
& E\left|L(\Phi)_{k}^{n}-L_{k}^{n} \Phi\right|^{2} \\
& =E \mid-\beta \int_{n \Delta t}^{(n+1) \Delta t} \Phi_{x x}(k \Delta x, s) d s \\
& +r \beta(2 \Phi((k-3) \Delta x, n \Delta t)-27 \Phi((k-2) \Delta x, n \Delta t)+270 \Phi((k-1) \Delta x, n \Delta t) \\
& -490 \Phi(k \Delta x, n \Delta t)+270 \Phi((k+1) \Delta x, n \Delta t)-27 \Phi((k+2) \Delta x, n \Delta t) \\
& +2 \Phi((k+3) \Delta x, n \Delta t))\left.\right|^{2}
\end{aligned}
$$




$$
\begin{gathered}
=E \mid-\beta\left[\int_{n \Delta t}^{(n+1) \Delta t} \Phi_{x x}(k \Delta x, s) d s\right. \\
-r(2 \Phi((k-3) \Delta x, n \Delta t)-27 \Phi((k-2) \Delta x, n \Delta t)+270 \Phi((k-1) \Delta x, n \Delta t) \\
-490 \Phi(k \Delta x, n \Delta t)+270 \Phi((k+1) \Delta x, n \Delta t)-27 \Phi((k+2) \Delta x, n \Delta t) \\
+2 \Phi((k+3) \Delta x, n \Delta t))]\left.\right|^{2}
\end{gathered}
$$

Hence:

$$
\begin{aligned}
& E\left|L(\Phi)_{k}^{n}-L_{k}^{n} \Phi\right|^{2} \leq E\left|\beta^{2}\right| \int_{n \Delta t}^{(n+1) \Delta t} \Phi_{x x}(k \Delta x, s) d s-\frac{k^{2}}{n} \frac{\mathrm{n} \Delta t}{180 k^{2} \Delta x^{2}}(2 \Phi((k- \\
& \text { 3) } \Delta x, n \Delta t)-27 \Phi((k-2) \Delta x, n \Delta t)+270 \Phi((k-1) \Delta x, n \Delta t)- \\
& 490 \Phi(k \Delta x, n \Delta t)+270 \Phi((k+1) \Delta x, n \Delta t)-27 \Phi((k+2) \Delta x, n \Delta t)+ \\
& 2 \Phi((k+3) \Delta x, n \Delta t))]^{2} \mid \\
& \text { As: } \quad \Delta \mathrm{t} \rightarrow 0, \Delta \mathrm{x} \rightarrow 0, \mathrm{n} \rightarrow \infty, \mathrm{k} \rightarrow \infty, \frac{k^{2}}{n} \rightarrow 0 \text { and }(\mathrm{k} \Delta \mathrm{x}, \mathrm{n} \Delta \mathrm{t}) \rightarrow(\mathrm{x}, \mathrm{t})
\end{aligned}
$$

Then

$$
E\left|L(\Phi)_{k}^{n}-L_{k}^{n} \Phi(k \Delta x, n \Delta t)\right|^{2} \rightarrow 0
$$

Hence, the random difference scheme (2) is consistent in mean square sense.

\subsection{Stability of RFDS (2)}

Theorem (2). The random difference scheme with seven points (2) satisfy the stability if $k=1$ and $b=0$.

\section{The proof:}

As we have:

$$
u_{k}^{n+1}=(1-490 r \beta) u_{k}^{n}+r \beta\left[2 u_{k-3}^{n}-27 u_{k-2}^{n}+270 u_{k-1}^{n}+270 u_{k+1}^{n}-27 u_{k+2}^{n}+2 u_{k+3}^{n}\right]
$$

Then:

$$
\begin{aligned}
\mathrm{E}\left|\mathrm{u}_{\mathrm{k}}^{\mathrm{n}+1}\right|^{2}=E \mid(1 & -490 r \beta) u_{k}^{n}+\left.r \beta\left[2 u_{k-3}^{n}-27 u_{k-2}^{n}+270 u_{k-1}^{n}+270 u_{k+1}^{n}-27 u_{k+2}^{n}+2 u_{k+3}^{n}\right]\right|^{2} \\
= & E \mid(1-490 r \beta)^{2}\left(u_{k}^{n}\right)^{2} \\
& +(r \beta)^{2}\left[2 u_{k-3}^{n}-27 u_{k-2}^{n}+270 u_{k-1}^{n}+270 u_{k+1}^{n}-27 u_{k+2}^{n}+2 u_{k+3}^{n}\right]^{2} \\
& +2 r \beta(1-490 r \beta)\left(u_{k}^{n}\right)\left[2 u_{k-3}^{n}-27 u_{k-2}^{n}+270 u_{k-1}^{n}+270 u_{k+1}^{n}-27 u_{k+2}^{n}+2 u_{k+3}^{n}\right] \mid
\end{aligned}
$$

Hence: 


$$
\begin{aligned}
& E\left|u_{k}^{n+1}\right|^{2}=E\left|u_{k}^{n}\right|^{2}-980 E\left|r \beta\left(u_{k}^{n}\right)^{2}\right|+240100 E\left|(r \beta)^{2}\left(u_{k}^{n}\right)^{2}\right| \\
&+ {\left[4 E\left|(r \beta)^{2}\left(u_{k-3}^{n}\right)^{2}\right|+8 E\left|(r \beta)^{2}\left(u_{k-3}^{n} u_{k+3}^{n}\right)\right|+4 E\left|(r \beta)^{2}\left(u_{k+3}^{n}\right)^{2}\right|+729 E\left|(r \beta)^{2}\left(u_{k-2}^{n}\right)^{2}\right|\right.} \\
&+1458 E\left|(r \beta)^{2}\left(u_{k-2}^{n} u_{k+2}^{n}\right)\right|+729 E\left|(r \beta)^{2}\left(u_{k+2}^{n}\right)^{2}\right|+72900 E\left|(r \beta)^{2}\left(u_{k-1}^{n}\right)^{2}\right| \\
&+145800 E\left|(r \beta)^{2}\left(u_{k-1}^{n} u_{k+1}^{n}\right)\right|+72900 E\left|(r \beta)^{2}\left(u_{k+1}^{n}\right)^{2}\right| \\
&-108\left[E\left|(r \beta)^{2}\left(u_{k-3}^{n} u_{k-2}^{n}\right)\right|+E\left|(r \beta)^{2}\left(u_{k-3}^{n} u_{k+2}^{n}\right)\right|+E\left|(r \beta)^{2}\left(u_{k+3}^{n} u_{k-2}^{n}\right)\right|\right. \\
&\left.+E\left|(r \beta)^{2}\left(u_{k+3}^{n} u_{k+2}^{n}\right)\right|\right] \\
&+1080\left[E\left|(r \beta)^{2}\left(u_{k-3}^{n} u_{k-1}^{n}\right)\right|+E\left|(r \beta)^{2}\left(u_{k-3}^{n} u_{k+1}^{n}\right)\right|+E\left|(r \beta)^{2}\left(u_{k+3}^{n} u_{k-1}^{n}\right)\right|\right. \\
&\left.+E\left|(r \beta)^{2}\left(u_{k+3}^{n} u_{k+1}^{n}\right)\right|\right] \\
&-14580\left[E\left|(r \beta)^{2}\left(u_{k-2}^{n} u_{k-1}^{n}\right)\right|+E\left|(r \beta)^{2}\left(u_{k-2}^{n} u_{k+1}^{n}\right)\right|+E\left|(r \beta)^{2}\left(u_{k+2}^{n} u_{k-1}^{n}\right)\right|\right. \\
&\left.\left.+E\left|(r \beta)^{2}\left(u_{k+2}^{n} u_{k+1}^{n}\right)\right|\right]\right]+4 E\left|(r \beta)\left(u_{k}^{n} u_{k-3}^{n}\right)\right|-1960 E\left|(r \beta)^{2}\left(u_{k}^{n} u_{k-3}^{n}\right)\right| \\
&-54 E\left|(r \beta)\left(u_{k}^{n} u_{k-2}^{n}\right)\right|+26460 E\left|(r \beta)^{2}\left(u_{k}^{n} u_{k-2}^{n}\right)\right|+540 E\left|(r \beta)\left(u_{k}^{n} u_{k-1}^{n}\right)\right| \\
&-264600 E\left|(r \beta)^{2}\left(u_{k}^{n} u_{k-1}^{n}\right)\right|+540 E\left|(r \beta)\left(u_{k}^{n} u_{k+1}^{n}\right)\right|-264600 E\left|(r \beta)^{2}\left(u_{k}^{n} u_{k+1}^{n}\right)\right| \\
&-54 E\left|(r \beta)\left(u_{k}^{n} u_{k-2}^{n}\right)\right|+26460 E\left|(r \beta)^{2}\left(u_{k}^{n} u_{k+2}^{n}\right)\right|+4 E\left|(r \beta)\left(u_{k}^{n} u_{k+3}^{n}\right)\right| \\
&-1960 E\left|(r \beta)^{2}\left(u_{k}^{n} u_{k+3}^{n}\right)\right|
\end{aligned}
$$

By using the supermom we have:

$$
\begin{aligned}
E\left|u_{k}^{n+1}\right|^{2} \leq \sup _{k}[ & E\left|u_{k}^{n}\right|^{2}-980 E\left|r \beta\left(u_{k}^{n}\right)^{2}\right|+240100 E\left|(r \beta)^{2}\left(u_{k}^{n}\right)^{2}\right| \\
& +\left[4 E\left|(r \beta)^{2}\left(u_{k-3}^{n}\right)^{2}\right|+8 E\left|(r \beta)^{2}\left(u_{k-3}^{n} u_{k+3}^{n}\right)\right|+4 E\left|(r \beta)^{2}\left(u_{k+3}^{n}\right)^{2}\right|+729 E\left|(r \beta)^{2}\left(u_{k-2}^{n}\right)^{2}\right|\right. \\
& +1458 E\left|(r \beta)^{2}\left(u_{k-2}^{n} u_{k+2}^{n}\right)\right|+729 E\left|(r \beta)^{2}\left(u_{k+2}^{n}\right)^{2}\right|+72900 E\left|(r \beta)^{2}\left(u_{k-1}^{n}\right)^{2}\right| \\
& +145800 E\left|(r \beta)^{2}\left(u_{k-1}^{n} u_{k+1}^{n}\right)\right|+72900 E\left|(r \beta)^{2}\left(u_{k+1}^{n}\right)^{2}\right| \\
& -108\left[E\left|(r \beta)^{2}\left(u_{k-3}^{n} u_{k-2}^{n}\right)\right|+E\left|(r \beta)^{2}\left(u_{k-3}^{n} u_{k+2}^{n}\right)\right|+E\left|(r \beta)^{2}\left(u_{k+3}^{n} u_{k-2}^{n}\right)\right|\right. \\
& \left.+E\left|(r \beta)^{2}\left(u_{k+3}^{n} u_{k+2}^{n}\right)\right|\right] \\
& +1080\left[E\left|(r \beta)^{2}\left(u_{k-3}^{n} u_{k-1}^{n}\right)\right|+E\left|(r \beta)^{2}\left(u_{k-3}^{n} u_{k+1}^{n}\right)\right|+E\left|(r \beta)^{2}\left(u_{k+3}^{n} u_{k-1}^{n}\right)\right|\right. \\
& \left.+E\left|(r \beta)^{2}\left(u_{k+3}^{n} u_{k+1}^{n}\right)\right|\right] \\
& -14580\left[E\left|(r \beta)^{2}\left(u_{k-2}^{n} u_{k-1}^{n}\right)\right|+E\left|(r \beta)^{2}\left(u_{k-2}^{n} u_{k+1}^{n}\right)\right|+E\left|(r \beta)^{2}\left(u_{k+2}^{n} u_{k-1}^{n}\right)\right|\right. \\
& \left.\left.+E\left|(r \beta)^{2}\left(u_{k+2}^{n} u_{k+1}^{n}\right)\right|\right]\right]+4 E\left|(r \beta)\left(u_{k}^{n} u_{k-3}^{n}\right)\right|-1960 E\left|(r \beta)^{2}\left(u_{k}^{n} u_{k-3}^{n}\right)\right| \\
& -54 E\left|(r \beta)\left(u_{k}^{n} u_{k-2}^{n}\right)\right|+26460 E\left|(r \beta)^{2}\left(u_{k}^{n} u_{k-2}^{n}\right)\right|+540 E\left|(r \beta)\left(u_{k}^{n} u_{k-1}^{n}\right)\right| \\
& -264600 E\left|(r \beta)^{2}\left(u_{k}^{n} u_{k-1}^{n}\right)\right|+540 E\left|(r \beta)\left(u_{k}^{n} u_{k+1}^{n}\right)\right|-264600 E\left|(r \beta)^{2}\left(u_{k}^{n} u_{k+1}^{n}\right)\right| \\
& -54 E\left|(r \beta)\left(u_{k}^{n} u_{k-2}^{n}\right)\right|+26460 E\left|(r \beta)^{2}\left(u_{k}^{n} u_{k+2}^{n}\right)\right|+4 E\left|(r \beta)\left(u_{k}^{n} u_{k+3}^{n}\right)\right| \\
& \left.-1960 E\left|(r \beta)^{2}\left(u_{k}^{n} u_{k+3}^{n}\right)\right|\right]
\end{aligned}
$$

Hence, 


$$
\begin{aligned}
& E\left|u_{k}^{n+1}\right|^{2} \leq \sup _{k} E\left|u_{k}^{n}\right|^{2}-980 \inf _{k} E\left|r \beta\left(u_{k}^{n}\right)^{2}\right|+240100 \sup _{k} E\left|(r \beta)^{2}\left(u_{k}^{n}\right)^{2}\right|+4 \sup _{k} E\left|(r \beta)^{2}\left(u_{k}^{n}\right)^{2}\right| \\
& +8 \sup _{k} E\left|(r \beta)^{2}\left(u_{k}^{n}\right)^{2}\right|+4 \sup _{k} E\left|(r \beta)^{2}\left(u_{k}^{n}\right)^{2}\right|+729 \sup _{k} E\left|(r \beta)^{2}\left(u_{k}^{n}\right)^{2}\right| \\
& +1458 \sup _{k} E\left|(r \beta)^{2}\left(u_{k}^{n}\right)^{2}\right|+729 \sup _{k} E\left|(r \beta)^{2}\left(u_{k}^{n}\right)^{2}\right|+72900 \sup _{k} E\left|(r \beta)^{2}\left(u_{k}^{n}\right)^{2}\right| \\
& +145800 \sup _{k} E\left|(r \beta)^{2}\left(u_{k}^{n}\right)^{2}\right|+72900 \sup _{k} E\left|(r \beta)^{2}\left(u_{k}^{n}\right)^{2}\right| \\
& -108\left[\inf _{k} E\left|(r \beta)^{2}\left(u_{k}^{n}\right)^{2}\right|+\inf _{k} E\left|(r \beta)^{2}\left(u_{k}^{n}\right)^{2}\right|+\inf _{k} E\left|(r \beta)^{2}\left(u_{k}^{n}\right)^{2}\right|+\inf _{k} E\left|(r \beta)^{2}\left(u_{k}^{n}\right)^{2}\right|\right] \\
& +1080\left[\sup _{k} E\left|(r \beta)^{2}\left(u_{k}^{n}\right)^{2}\right|+\sup _{k} E\left|(r \beta)^{2}\left(u_{k}^{n}\right)^{2}\right|+\sup _{k} E\left|(r \beta)^{2}\left(u_{k}^{n}\right)^{2}\right|\right. \\
& \left.+\sup _{k} E\left|(r \beta)^{2}\left(u_{k}^{n}\right)^{2}\right|\right] \\
& -14580\left[\inf _{k} E\left|(r \beta)^{2}\left(u_{k}^{n}\right)^{2}\right|+\inf _{k} E\left|(r \beta)^{2}\left(u_{k}^{n}\right)^{2}\right|+\inf _{k} E\left|(r \beta)^{2}\left(u_{k}^{n}\right)^{2}\right|\right. \\
& \left.+\inf _{k} E\left|(r \beta)^{2}\left(u_{k}^{n}\right)^{2}\right|\right]+4 \sup _{k} E\left|(r \beta)\left(u_{k}^{n}\right)^{2}\right|-1960 \inf _{k} E\left|(r \beta)^{2}\left(u_{k}^{n}\right)^{2}\right| \\
& -54 \inf _{k} E\left|(r \beta)\left(u_{k}^{n}\right)^{2}\right|+26460 \sup _{k} E\left|(r \beta)^{2}\left(u_{k}^{n}\right)^{2}\right|+540 \sup _{k} E\left|(r \beta)\left(u_{k}^{n}\right)^{2}\right| \\
& -264600 \inf _{k} E\left|(r \beta)^{2}\left(u_{k}^{n}\right)^{2}\right|+540 \sup _{k} E\left|(r \beta)\left(u_{k}^{n}\right)^{2}\right|-264600 \inf _{k} E\left|(r \beta)^{2}\left(u_{k}^{n}\right)^{2}\right| \\
& -54 \inf _{k} E\left|(r \beta)\left(u_{k}^{n}\right)^{2}\right|+26460 \sup _{k} E\left|(r \beta)^{2}\left(u_{k}^{n}\right)^{2}\right|+4 \sup _{k} E\left|(r \beta)\left(u_{k}^{n}\right)^{2}\right| \\
& -1960 \inf _{k} E\left|(r \beta)^{2}\left(u_{k}^{n}\right)^{2}\right| .
\end{aligned}
$$

As: $r \rightarrow 0$ and for large $k$ and $n$ we can deduce that:

$$
E\left|u_{k}^{n+1}\right|^{2} \leq \sup _{k} E\left|u_{k}^{n}\right|^{2}
$$

\section{Hence:}

Then:

$$
\sup _{k} E\left|u_{k}^{n+1}\right|^{2} \leq \sup _{k} E\left|u_{k}^{n}\right|^{2} \leq \sup _{k} E\left|u_{k}^{n-1}\right|^{2} \leq \cdots \leq \sup _{k} E\left|u_{k}^{0}\right|^{2}
$$

$$
E\left|u^{n+1}\right|^{2} \leq E\left|u^{0}\right|^{2}
$$

Where $K=1$, and $b=0$, then the random scheme (2) satisfy the stability.

\subsection{Convergence of RFDS (2)}

Theorem (3). The random difference scheme with seven points (2) satisfy the convergence if:

$$
k \rightarrow \infty, n \rightarrow \infty, \Delta x \rightarrow 0, \Delta t \rightarrow 0, \frac{k^{2}}{n} \rightarrow 0 \text { and }(k \Delta x, n \Delta t) \rightarrow(x, t)
$$




\section{The proof}

$$
E\left|u_{k}^{n}-u\right|^{2}=E\left|\left(L_{k}^{n}\right)^{-1}\left(L_{k}^{n} u_{k}^{n}-L_{k}^{n} u\right)\right|^{2}
$$

As we proved that scheme (2) is consistent, thus:

$$
L_{k}^{n} u_{k}^{n} \stackrel{m . s .}{\longrightarrow} L u
$$

Hence we obtain:

$$
E\left|\left(L_{k}^{n} u_{k}^{n}-L_{k}^{n} u\right)\right|^{2} \rightarrow 0
$$

At: as $\Delta t \rightarrow 0, \Delta x \rightarrow 0, n \rightarrow \infty, k \rightarrow \infty$ and $(\mathrm{k} \Delta \mathrm{x}, \mathrm{n} \Delta \mathrm{t}) \rightarrow(\mathrm{x}, \mathrm{t})^{\prime}$

Since the scheme is stable that mean $\left(\mathrm{L}_{\mathrm{k}}^{\mathrm{n}}\right)^{-1}$ is bounded

Hence:

$$
E\left|u_{k}^{n}-u\right|^{2} \rightarrow 0
$$

As: $\Delta t \rightarrow 0, \Delta x \rightarrow 0, n \rightarrow \infty, k \rightarrow \infty, \frac{k^{2}}{n} \rightarrow 0$ and $(k \Delta x, n \Delta t) \rightarrow(x, t)$

Therefore, the scheme (2) has been convergent.

\section{Case study}

$$
\begin{aligned}
u_{t}=\beta u_{x x}(x, t), t \in[0, T], x \in[0, X], \beta(r . v .) & (3) \\
& u(x, 0)=\sin (\pi x) \\
& u(0, t)=u(X, t)=0
\end{aligned}
$$

4.1The Analytical Solution

$$
u(x, t)=e^{-\beta \pi^{2} t} \sin (\pi x)
$$

4.2 The Numerical Relation by Using RFDS ('with three and five points)

With three points:

$$
u_{k}^{n}=\sin (k \Delta x \pi)\left[1-2 \frac{\beta \Delta t}{(\Delta x)^{2}}(1-\cos (\Delta x \pi))\right]^{n}
$$

With five points:

$$
u_{k}^{n}=\sin (k \Delta x \pi)\left[1-\frac{7 \beta \Delta t}{3(\Delta x)^{2}}-\frac{1 \beta \Delta t}{3(\Delta x)^{2}} \cos (\Delta x \pi)(\cos (\Delta x \pi)-8)\right]^{n}
$$

\subsection{The Numerical Relation by Using RFDS (with seven points)}

Here we have the scheme,

$$
\begin{gathered}
u_{k}^{n+1}=(1-490 r \beta) u_{k}^{n}+r \beta\left[2 u_{k-3}^{n}-27 u_{k-2}^{n}+270 u_{k-1}^{n}+270 u_{k+1}^{n}-27 u_{k+2}^{n}+2 u_{k+3}^{n}\right] \\
u_{k}^{0}=\sin \left(\pi x_{k}\right), \\
u_{0}^{n}=u_{x}^{n}=0,
\end{gathered}
$$

As: $=\frac{\Delta t}{180 \Delta x^{2}}, \mathrm{x}_{\mathrm{k}}=k \Delta x, t_{n}=n \Delta t, \beta$ is a random variable.

First from the initial condition. we have:

$$
u_{k}^{0}=\sin (k \Delta x \pi)
$$




$$
\begin{aligned}
& u_{k}^{1}=(1-490 r) u_{k}^{0}+r\left(2\left(u_{k+3}^{0}+u_{k-3}^{0}\right)-27\left(u_{k+2}^{0}+u_{k-2}^{0}\right)+270\left(u_{k+1}^{0}+u_{k-1}^{0}\right)\right) \\
& =(1-490 r) \sin (k \Delta x \pi) \\
& +r(2(\sin ((k+3) \Delta x \pi)+\sin ((k-3) \Delta x \pi))-27(\sin ((k+2) \Delta x \pi)+\sin ((k-2) \Delta x \pi)) \\
& +270(\sin ((k+1) \Delta x \pi)+\sin ((k-1) \Delta x \pi))) \\
& =(1-490 r) u_{k}^{0}+r\left(2\left(u_{k+3}^{0}+u_{k-3}^{0}\right)-27\left(u_{k+2}^{0}+u_{k-2}^{0}\right)+270\left(u_{k+1}^{0}+u_{k-1}^{0}\right)\right) \\
& =(1-490 r) \sin (k \Delta x \pi) \\
& +r(2(2 \sin (k \Delta x \pi) \cos (3 \Delta x \pi))-27(2 \sin (k \Delta x \pi) \cos (2 \Delta x \pi)) \\
& +270(2 \sin (k \Delta x \pi) \cos (\Delta x \pi))) \\
& =\sin (k \Delta x \pi)[1-490 r+4 r \cos (3 \Delta x \pi)-54 r \cos (2 \Delta x \pi)+540 r \cos (\Delta x \pi)] \\
& =\sin (k \Delta x \pi)\left[1-490 r+4 r \cos (3 \Delta x \pi)-54 r\left(2 \cos ^{2}(\Delta x \pi)-1\right)+540 r \cos (\Delta x \pi)\right] \\
& =\sin (k \Delta x \pi)\left[1-490 r+4 r \cos (3 \Delta x \pi)-108 r \cos ^{2}(\Delta x \pi)+54 r+540 r \cos (\Delta x \pi)\right] \\
& =\sin (k \Delta x \pi)[1-436 r+4 r \cos (3 \Delta x \pi)-108 r \cos (\Delta x \pi)(\cos (\Delta x \pi)-5)] u_{k}^{2} \\
& =(1-490 r) u_{k}^{1}+r\left(2\left(u_{k+3}^{1}+u_{k-3}^{1}\right)-27\left(u_{k+2}^{1}+u_{k-2}^{1}\right)+270\left(u_{k+1}^{1}+u_{k-1}^{1}\right)\right) \\
& =(1-490 r) \sin (k \Delta x \pi)[1-436 r+4 r \cos (3 \Delta x \pi)-108 r \cos (\Delta x \pi)(\cos (\Delta x \pi)-5)] \\
& +r(2(\sin ((k+3) \Delta x \pi)[1-436 r+4 r \cos (3 \Delta x \pi)-108 r \cos (\Delta x \pi)(\cos (\Delta x \pi)-5)] \\
& +\sin ((k-3) \Delta x \pi)[1-436 r+4 r \cos (3 \Delta x \pi)-108 r \cos (\Delta x \pi)(\cos (\Delta x \pi)-5)]) \\
& -27(\sin ((k+2) \Delta x \pi)[1-436 r+4 r \cos (3 \Delta x \pi)-108 r \cos (\Delta x \pi)(\cos (\Delta x \pi)-5)] \\
& +\sin ((k-2) \Delta x \pi)[1-436 r+4 r \cos (3 \Delta x \pi)-108 r \cos (\Delta x \pi)(\cos (\Delta x \pi)-5)]) \\
& +270(\sin ((k+1) \Delta x \pi)[1-436 r+4 r \cos (3 \Delta x \pi)-108 r \cos (\Delta x \pi)(\cos (\Delta x \pi)-5)] \\
& +\sin ((k-1) \Delta x \pi)[1-436 r+4 r \cos (3 \Delta x \pi)-108 r \cos (\Delta x \pi)(\cos (\Delta x \pi)-5)])) \\
& =[1-436 r+4 r \cos (3 \Delta x \pi)-108 r \cos (\Delta x \pi)(\cos (\Delta x \pi)-5)][(1-490 r) \sin (k \Delta x \pi) \\
& ++r(2(\sin ((k+3) \Delta x \pi)+\sin ((k-3) \Delta x \pi))-27(\sin ((k+2) \Delta x \pi)+\sin ((k-2) \Delta x \pi)) \\
& +270(\sin ((k+1) \Delta x \pi)+\sin ((k-1) \Delta x \pi)))] \\
& =\sin (k \Delta x \pi)[1-436 r+4 r \cos (3 \Delta x \pi)-108 r \cos (\Delta x \pi)(\cos (\Delta x \pi)-5)]^{2}
\end{aligned}
$$




$$
\begin{aligned}
u_{k}^{3}=(1-490 r) & u_{k}^{2}+r\left(2\left(u_{k+3}^{2}+u_{k-3}^{2}\right)-27\left(u_{k+2}^{2}+u_{k-2}^{2}\right)+270\left(u_{k+1}^{2}+u_{k-1}^{2}\right)\right) \\
& =(-490 r) \sin (k \Delta x \pi)[1-436 r+4 r \cos (3 \Delta x \pi)-108 r \cos (\Delta x \pi)(\cos (\Delta x \pi)-5)]^{2} \\
& +r\left(2 \left(\sin ((k+3) \Delta x \pi)[1-436 r+4 r \cos (3 \Delta x \pi)-108 r \cos (\Delta x \pi)(\cos (\Delta x \pi)-5)]^{2}\right.\right. \\
& \left.+\sin ((k-3) \Delta x \pi)[1-436 r+4 r \cos (3 \Delta x \pi)-108 r \cos (\Delta x \pi)(\cos (\Delta x \pi)-5)]^{2}\right) \\
& -27\left(\sin ((k+2) \Delta x \pi)[1-436 r+4 r \cos (3 \Delta x \pi)-108 r \cos (\Delta x \pi)(\cos (\Delta x \pi)-5)]^{2}\right. \\
& \left.+\sin ((k-2) \Delta x \pi)[1-436 r+4 r \cos (3 \Delta x \pi)-108 r \cos (\Delta x \pi)(\cos (\Delta x \pi)-5)]^{2}\right) \\
& +270\left(\sin ((k+1) \Delta x \pi)[1-436 r+4 r \cos (3 \Delta x \pi)-108 r \cos (\Delta x \pi)(\cos (\Delta x \pi)-5)]^{2}\right. \\
& \left.\left.+\sin ((k-1) \Delta x \pi)[1-436 r+4 r \cos (3 \Delta x \pi)-108 r \cos (\Delta x \pi)(\cos (\Delta x \pi)-5)]^{2}\right)\right) \\
& =[1-436 r+4 r \cos (3 \Delta x \pi)-108 r \cos (\Delta x \pi)(\cos (\Delta x \pi)-5)]^{2}[(1-490 r) \sin (k \Delta x \pi) \\
& +r(2(\sin ((k+3) \Delta x \pi)+\sin ((k-3) \Delta x \pi))-27(\sin ((k+2) \Delta x \pi)+\sin ((k-2) \Delta x \pi)) \\
& +270(\sin ((k+1) \Delta x \pi)+\sin ((k-1) \Delta x \pi)))] \\
& =\sin (k \Delta x \pi)[1-436 r+4 r \cos (3 \Delta x \pi)-108 r \cos (\Delta x \pi)(\cos (\Delta x \pi)-5)]^{3}
\end{aligned}
$$

Then we have:

$$
u_{k}^{n}=\sin (k \Delta x \pi)[1-436 r+4 r \cos (3 \Delta x \pi)-108 r \cos (\Delta x \pi)(\cos (\Delta x \pi)-5)]^{n}
$$

Finally:

$$
u_{k}^{n}=\sin (k \Delta x \pi)\left[1-\frac{109 \beta \Delta t}{45(\Delta x)^{2}}+\frac{\beta \Delta t}{45(\Delta x)^{2}} \cos (3 \Delta x \pi)-\frac{3 \beta \Delta t}{5(\Delta x)^{2}} \cos (\Delta x \pi)(\cos (\Delta x \pi)-5)\right]^{n}
$$


Finite Difference Scheme for Solving Parabolic Partial Differential Equations with Random Variable Input 271 under Mean Square Sense

1. With three points

$$
\beta \sim \operatorname{Poisson}(0.5)
$$

\begin{tabular}{|c|c|c|c|c|c|c|}
\hline$k$ & $n$ & $x_{k}$ & $t_{n}$ & $E\left(u_{k}^{n}\right)$ & $E\left(u(x, t)_{x_{k}, t_{n}}\right)$ & $E\left(u(x, t)_{x_{k}, t_{n}}\right)-E\left(u_{k}^{n}\right)$ \\
\hline 1 & 1 & 0.1 & 0.005 & 0.3014548103 & 0.3016662209 & 0.0002114106000 \\
\hline 1 & 1 & 0.1 & 0.001 & 0.3075045577 & 0.3075032768 & 0.000001280900000 \\
\hline 1 & 1 & 0.1 & 0.000001 & 0.3090154821 & 0.3090154694 & $1.270000000 \times 10^{-8}$ \\
\hline 1 & 1 & 0.1 & 0.0000001 & 0.3090168433 & 0.3090168419 & $1.400000000 \times 10^{-9}$ \\
\hline
\end{tabular}

2. With five points

\begin{tabular}{|c|c|c|c|c|c|c|}
\hline$k$ & $n$ & $x_{k}$ & $t_{n}$ & $E\left(u_{k}^{n}\right)$ & $E\left(u(x, t)_{x_{k}, t_{n}}\right)$ & $E\left(u(x, t)_{x_{k}, t_{n}}\right)-E\left(u_{k}^{n}\right)$ \\
\hline 1 & 1 & 0.1 & 0.005 & 0.3013931238 & 0.3016662209 & 0.0002730971000 \\
\hline 1 & 1 & 0.1 & 0.001 & 0.3074922202 & 0.3075032768 & 0.00001105660000 \\
\hline 1 & 1 & 0.1 & 0.000001 & 0.3090154696 & 0.3090154694 & $2.000000000 \times 10^{-10}$ \\
\hline 1 & 1 & 0.1 & 0.0000001 & 0.3090168418 & 0.3090168419 & $1.000000000 \times 10^{-10}$ \\
\hline
\end{tabular}

3 . with seven points

\begin{tabular}{|l|l|l|l|l|l|l|}
\hline$k$ & $n$ & $x_{k}$ & $t_{n}$ & $E\left(u_{k}^{n}\right)$ & $E\left(u(x, t)_{x_{k}, t_{n}}\right)$ & $E\left(u(x, t)_{x_{k}, t_{n}}\right)-E\left(u_{k}^{n}\right)$ \\
\hline 1 & 1 & 0.1 & 0.005 & 0.3013923186 & 0.3016662209 & 0.0002739023000 \\
\hline 1 & 1 & 0.1 & 0.001 & 0.3074920592 & 0.3075032768 & 0.00001121760000 \\
\hline 1 & 1 & 0.1 & 0.000001 & 0.3090154695 & 0.3090154694 & $1.000000000 \times 10^{-10}$ \\
\hline 1 & 1 & 0.1 & 0.0000001 & 0.3090168419 & 0.3090168419 & 0. \\
\hline
\end{tabular}
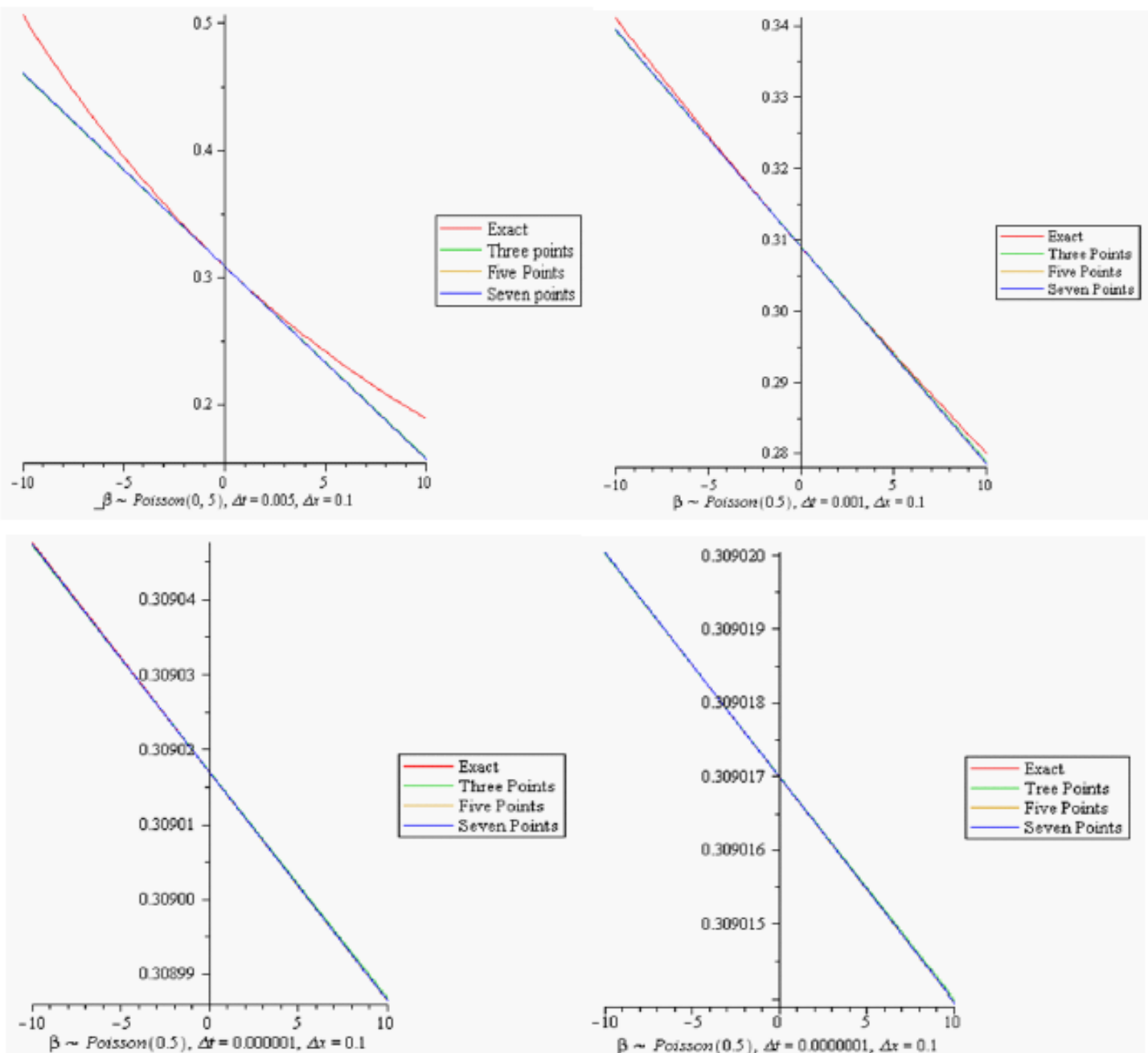

Fig. 1 

under Mean Square Sense

$$
\beta \sim \operatorname{Poisson}(0.25)
$$

4. with three points

\begin{tabular}{|c|c|c|c|c|c|c|}
\hline$k$ & $n$ & $x_{k}$ & $t_{n}$ & $E\left(u_{k}^{n}\right)$ & $E\left(u(x, t)_{x_{k}, t_{n}}\right)$ & $E\left(u(x, t)_{x_{k}, t_{n}}\right)-E\left(u_{k}^{n}\right)$ \\
\hline 1 & 1 & 0.1 & 0.005 & 0.3052359023 & 0.3053194865 & 0.00008358420000 \\
\hline 1 & 1 & 0.1 & 0.001 & 0.3082607760 & 0.3082592064 & 0.000001569600000 \\
\hline 1 & 1 & 0.1 & 0.000001 & 0.3090094323 & 0.3090162318 & $6.600000000 \times 10^{-9}$ \\
\hline 1 & 1 & 0.1 & 0.0000001 & 0.3090162384 & 0.3090169182 & $7.000000000 \times 10^{-10}$ \\
\hline
\end{tabular}

5. with five points

\begin{tabular}{|c|c|c|c|c|c|c|}
\hline$k$ & $n$ & $x_{k}$ & $t_{n}$ & $E\left(u_{k}^{n}\right)$ & $E\left(u(x, t)_{x_{k}, t_{n}}\right)$ & $E\left(u(x, t)_{x_{k}, t_{n}}\right)-E\left(u_{k}^{n}\right)$ \\
\hline 1 & 1 & 0.1 & 0.005 & 0.3052050592 & 0.3053194865 & 0.0001144273000 \\
\hline 1 & 1 & 0.1 & 0.001 & 0.3082546072 & 0.3082592064 & 0.000004599200000 \\
\hline 1 & 1 & 0.1 & 0.000001 & 0.3090162320 & 0.3090162318 & $2.000000000 \times 10^{-10}$ \\
\hline 1 & 1 & 0.1 & 0.0000001 & 0.3090169182 & 0.3090169182 & 0 . \\
\hline
\end{tabular}
6 . with seven points

\begin{tabular}{|c|c|c|c|c|c|c|}
\hline$k$ & $n$ & $x_{k}$ & $t_{n}$ & $E\left(u_{k}^{n}\right)$ & $E\left(u(x, t)_{x_{n}, t_{n}}\right)$ & $E\left(u(x, t)_{x_{k}, t_{n}}\right)-E\left(u_{k}^{n}\right)$ \\
\hline 1 & 1 & 0.1 & 0.005 & 0.3052046565 & 0.3053194865 & 0.0001148300000 \\
\hline 1 & 1 & 0.1 & 0.001 & 0.3082545268 & 0.3082592064 & 0.000004679600000 \\
\hline 1 & 1 & 0.1 & 0.000001 & 0.3090162321 & 0.3090162318 & $3.000000000 \times 10^{-10}$ \\
\hline 1 & 1 & 0.1 & 0.0000001 & 0.3090169183 & 0.3090169182 & $1.000000000 \times 10^{-10}$ \\
\hline
\end{tabular}
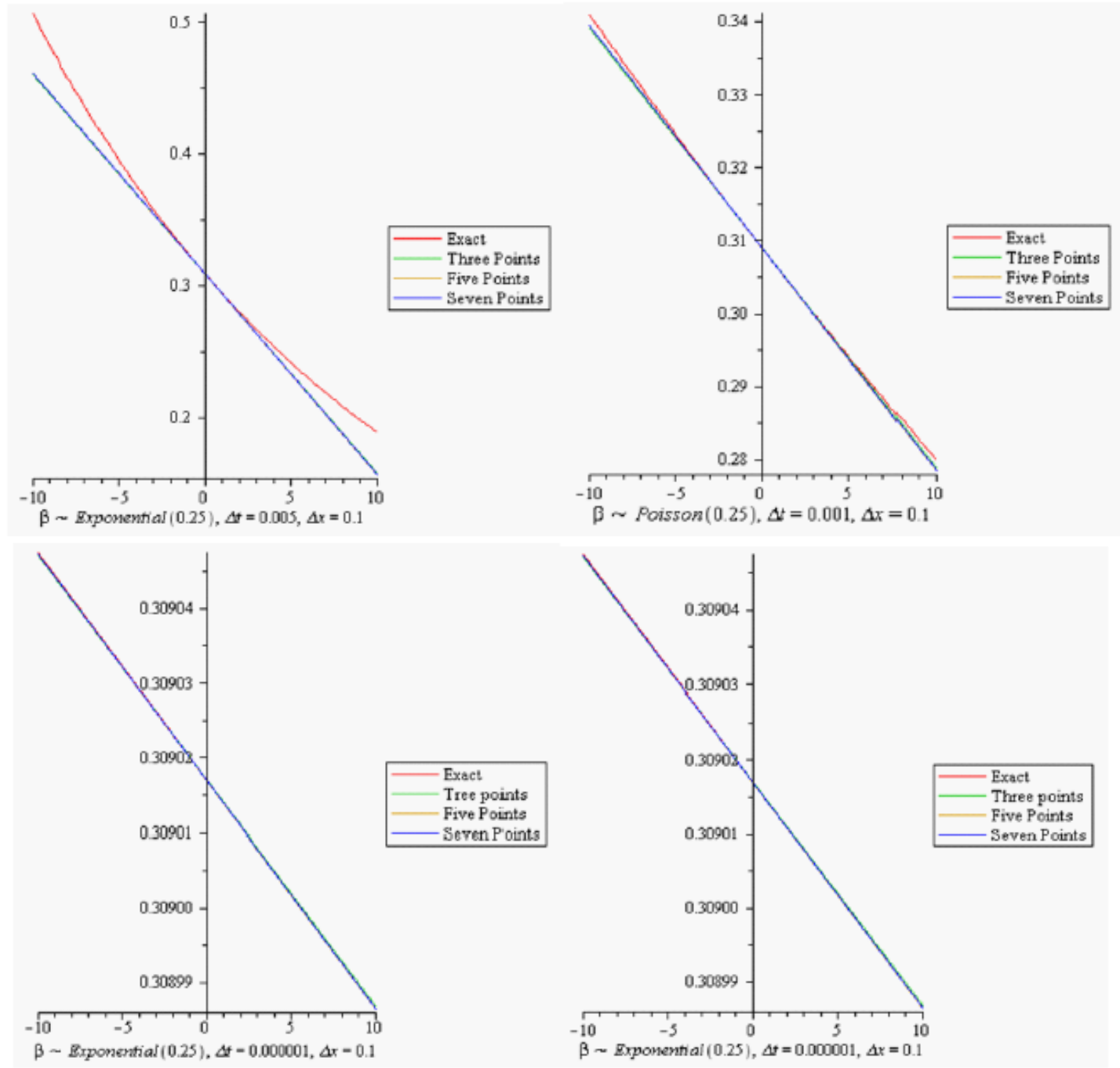

Fig. 2 
Finite Difference Scheme for Solving Parabolic Partial Differential Equations with Random Variable Input 273 under Mean Square Sense

$$
\beta \sim \operatorname{Poisson}(0.025)
$$

7. With three points

\begin{tabular}{|c|c|c|c|c|c|c|}
\hline$k$ & $n$ & $x_{k}$ & $t_{n}$ & $E\left(u_{k}^{n}\right)$ & $E\left(u(x, t)_{x_{k}, t_{n}}\right.$ & $E\left(u(x, t)_{x_{k}, t_{n}}\right)-E\left(u_{k}^{n}\right)$ \\
\hline 1 & 1 & 0.1 & 0.005 & 0.3086388854 & 0.3086452375 & 0.000006352100000 \\
\hline 1 & 1 & 0.1 & 0.001 & 0.3089413727 & 0.3089411317 & $2.410000000 \times 10^{-7}$ \\
\hline 1 & 1 & 0.1 & 0.000001 & 0.3090169188 & 0.3090169183 & $5.000000000 \times 10^{-10}$ \\
\hline 1 & 1 & 0.1 & 0.0000001 & 0.3090169870 & 0.3090169867 & $3.000000000 \times 10^{-10}$ \\
\hline
\end{tabular}

8. With five points

\begin{tabular}{|c|c|c|c|c|c|c|}
\hline$k$ & $n$ & $x_{k}$ & $t_{n}$ & $E\left(u_{k}^{n}\right)$ & $E\left(u(x, t)_{x_{k}, t_{n}}\right)$ & $E\left(u(x, t)_{x_{k}, t_{n}}\right)-E\left(u_{k}^{n}\right)$ \\
\hline 1 & 1 & 0.1 & 0.005 & 0.3086358010 & 0.3086452375 & 0.000009436500000 \\
\hline 1 & 1 & 0.1 & 0.001 & 0.3089407557 & 0.3089411317 & $3.760000000 \times 10^{-7}$ \\
\hline 1 & 1 & 0.1 & 0.000001 & 0.3090169183 & 0.3090169183 & 0. \\
\hline 1 & 1 & 0.1 & 0.0000001 & 0.3090169867 & 0.3090169867 & 0. \\
\hline
\end{tabular}

9. With seven points

\begin{tabular}{|c|c|c|c|c|c|c|}
\hline$k$ & $n$ & $x_{k}$ & $t_{n}$ & $E\left(u_{k}^{n}\right)$ & $E\left(u(x, t)_{x_{k}, t_{n}}\right)$ & $E\left(u(x, t)_{x_{k}, t_{n}}\right)-E\left(u_{k}^{n}\right)$ \\
\hline 1 & 1 & 0.1 & 0.005 & 0.3086357607 & 0.3086452375 & 0.000009476800000 \\
\hline 1 & 1 & 0.1 & 0.001 & 0.3089407479 & 0.3089411317 & $3.838000000 \times 10^{-7}$ \\
\hline 1 & 1 & 0.1 & 0.000001 & 0.3090169183 & 0.3090169183 & 0. \\
\hline 1 & 1 & 0.1 & 0.0000001 & 0.3090169870 & 0.3090169867 & $3.000000000 \times 10^{-10}$ \\
\hline
\end{tabular}
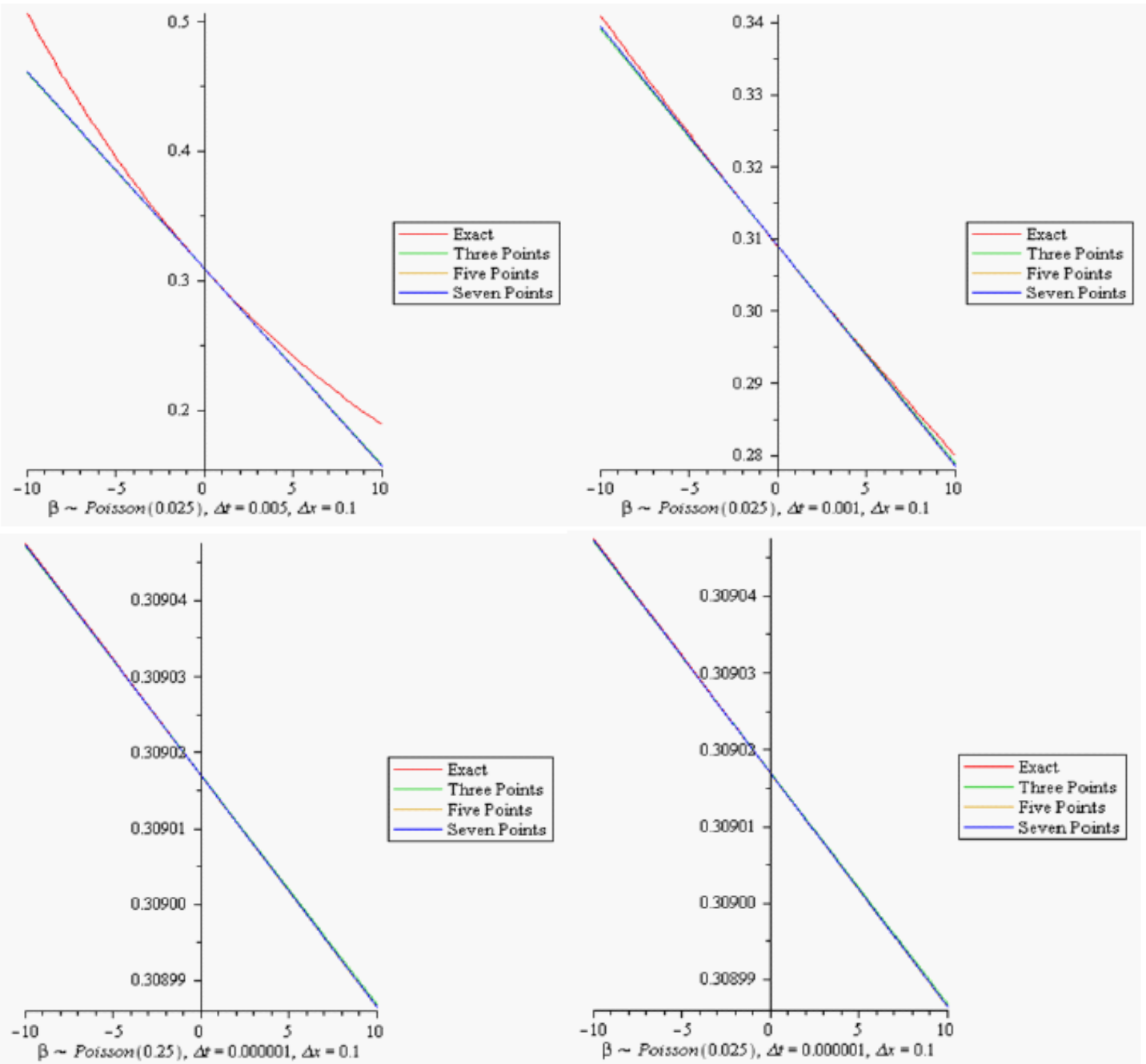

Fig. 3 

under Mean Square Sense

\section{$\beta \sim \operatorname{Exponential}(0.25)$}

10. with three points

\begin{tabular}{|c|c|c|c|c|c|c|}
\hline$k$ & $n$ & $x_{k}$ & $t_{n}$ & $E\left(u_{k}^{n}\right)$ & $E\left(u(x, t)_{x_{k}, t_{n}}\right)$ & $E\left(u(x, t)_{x_{k}, t_{n}}\right)-E\left(u_{k}^{n}\right)$ \\
\hline 1 & 1 & 0.1 & 0.005 & 0.3052359023 & 0.3052511098 & 0.00001520750000 \\
\hline 1 & 1 & 0.1 & 0.000001 & 0.3090162382 & 0.3090162319 & $6.300000000 \times 10^{-9}$ \\
\hline 1 & 1 & 0.1 & 0.0000001 & 0.3090169188 & 0.3090169182 & $6.000000000 \times 10^{-10}$ \\
\hline
\end{tabular}

11. with five points

\begin{tabular}{|c|c|c|c|c|c|c|}
\hline$k$ & $n$ & $x_{k}$ & $t_{n}$ & $E\left(u_{k}^{n}\right)$ & $E\left(u(x, t)_{x_{k}, t_{n}}\right)$ & $E\left(u(x, t)_{x_{k}, t_{n}}\right)-E\left(u_{k}^{n}\right)$ \\
\hline 1 & 1 & 0.1 & 0.005 & 0.3052050591 & 0.3052511098 & 0.00004605070000 \\
\hline 1 & 1 & 0.1 & 0.000001 & 0.3090162320 & 0.3090162319 & $1.000000000 \times 10^{-10}$ \\
\hline 1 & 1 & 0.1 & 0.0000001 & 0.3090169182 & 0.3090169182 & 0 . \\
\hline
\end{tabular}

12. with five points

\begin{tabular}{|c|c|c|c|c|c|c|}
\hline$k$ & $n$ & $x_{k}$ & $t_{n}$ & $E\left(u_{k}^{n}\right)$ & $E\left(u(x, t)_{x_{k}, t_{n}}\right)$ & $E\left(u(x, t)_{x_{k}, t_{n}}\right)-E\left(u_{k}^{n}\right)$ \\
\hline 1 & 1 & 0.1 & 0.005 & 0.3052046565 & 0.3052511098 & 0.00004645330000 \\
\hline 1 & 1 & 0.1 & 0.001 & 0.3082607760 & 0.3082564022 & 0.000004373800000 \\
\hline 1 & 1 & 0.1 & 0.000001 & 0.3090162319 & 0.3090162319 & 0 . \\
\hline 1 & 1 & 0.1 & 0.0000001 & 0.3090169182 & 0.3090169182 & 0. \\
\hline
\end{tabular}
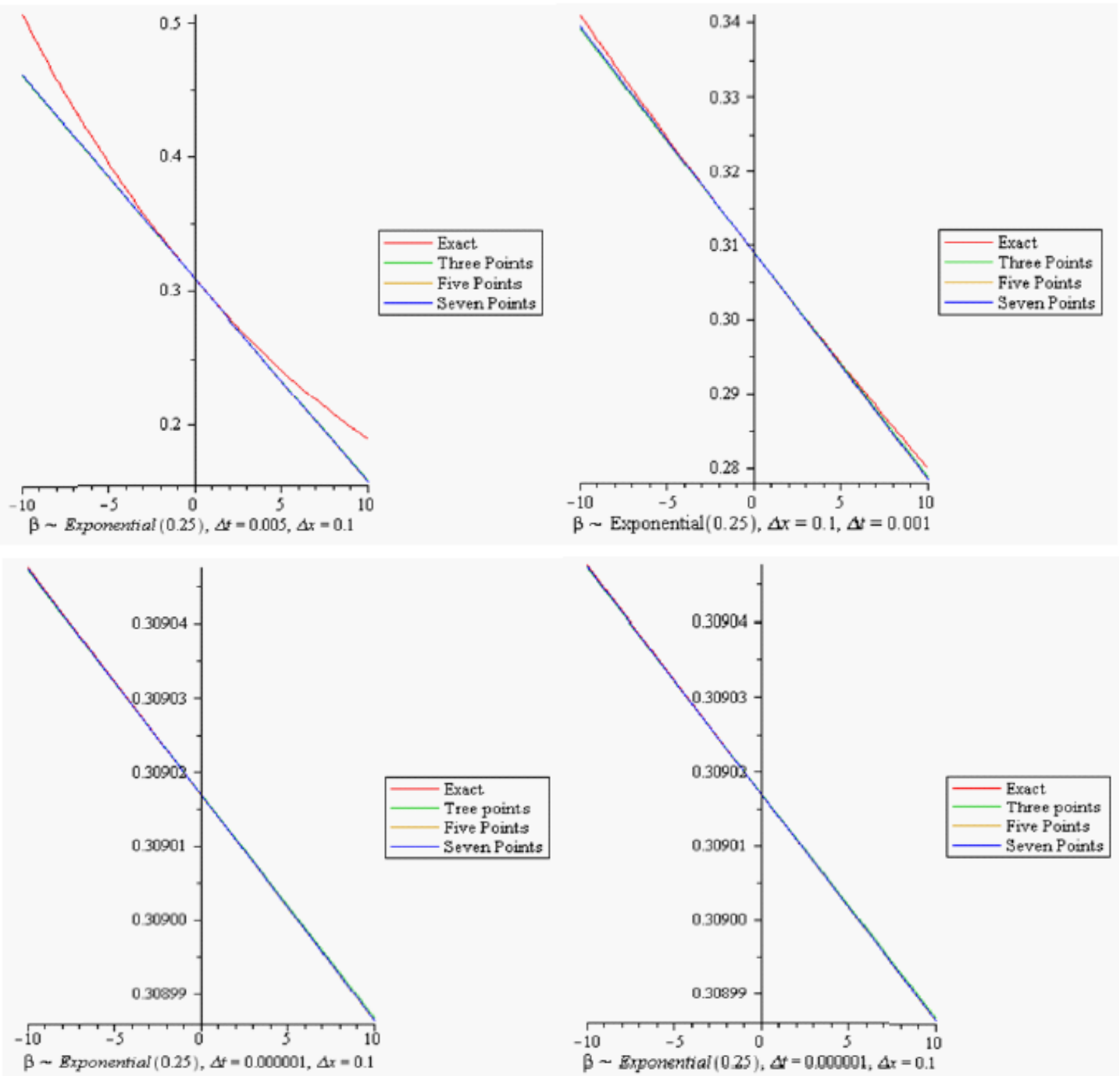

Fig. 4 

under Mean Square Sense

\section{Conclusion}

We have discussed the random problem by finite difference technique with applying the mean square basics.

\section{References}

[1] C. Roth, approximations of solution of a first order stochastic partial differential equation, Report, Institute Optimierung und Stochastic, Universitat Halle-wittenberg, Halle, (2001).

[2] J. Thomas, Numerical Partial Differential Equations: Finite Difference methods, Texts in Applied Mathematics, Springer, (1998).'

[3] P. E. Kloeden, E. Platen, Numerical Solution of Stochastic Differential Equations, Springer-Verlag, Berlin, (1992).

[4] T. T. Soong, Random Differential Equations in Science and Engineering, Academic Press, New York, (1973).

[5] T. Zhang. Numerical approximations of stochastic partial differential equations, M.Phil., thesis, Hong Kong
University of Science and Technology, Hong Kong, (2000).

[6] W. W. Mohammed, M. A. Sohaly, A. El-Bassiouny, and K. Elnagar. Mean Square Convergent Finite Difference Scheme for Stochastic Parabolic PDEs. American Journal of Computational Mathematics, 4:280-288 (2014).।

[7] M. A. Sohaly. Mean Square Convergent Three and Five Points Finite Difference Scheme for Stochastic Parabolic Partial Differential Equations, 2(1): 164-171 (2014).

[8] M. A. El-Tawil and M. A. Sohaly. Mean Square Numerical Methods for initial value random differential equations, open Journal of Discrete Mathematics OJDM, (1), 66-84 (2009).

[9] M. A. El-Tawil and M. A. Sohaly. Mean Square Convergent three points finite difference scheme for random partial differential equations, Journal of the Egyptian Mathematical Society, 20(3): 188-204 (2012).

[10] M. A. Sohaly. Mean Square Heun's Method Convergent for Solving Random Differential Initial Value Problems of First Order American Journal of Computational Mathematics, 4, 280-288 (2014). 